\title{
Novel Photometric Stereo Based Pulmonary Function Testing
}

\author{
Jahanzeb AHMAD*a ${ }^{* a}$ Jiuai SUN ${ }^{a}$, Lyndon SMITH ${ }^{a}$, Melvyn SMITH ${ }^{a}$, John HENDERSON ${ }^{b}$, \\ Anirban MAJUMDAR ${ }^{\mathrm{C}}$ \\ ${ }^{a}$ Machine Vision Laboratory, University of the West of England, Bristol, UK; \\ ${ }^{\mathrm{b}} \mathrm{S}$ chool of Social and Community Medicine, Bristol University, Bristol, UK; \\ ${ }^{\mathrm{c} B r i s t o l}$ Children's Hospital, Frenchay Hospital, Bristol, UK
}

\begin{abstract}
A Photometric stereo based unique and novel non-contact method has been developed to perform pulmonary functional testing. This system is based on two digital cameras and eight light sources. One camera observes the subject from the front and second from the back while subject is standing in the middle of rig wearing a tight white t-shirt. Registration between both sides of the rig is not required and light source direction calibration is only required once. The system can capture images of 1.4 Mega pixels at 110 frames per second (fps) using an ordinary desktop system and process it offline. Chest and abdomen wall motion is observed over time by estimating change in surface gradient calculated from Photometric Stereo. Very high (0.98) correlation between resulting volume from Photometric Stereo and Spirometer data is observed.
\end{abstract}

Keywords: photometric stereo, pulmonary functional testing

\section{Introduction}

Pulmonary Functional testing (PFT) are the most common test performed on patients to assess respiratory functionality. The devices commonly used to do Pulmonary Functional testing are Spirometer and Plethysmography. These devices are used to measure flow and volume of air inspired and expired from the lungs. Both devices are contact based invasive devices. The contact based nature of the devices increases breath awareness which may cause hyperventilation and alter the breathing pattern $[1,2]$ consequently a non contact device can reduce this problem. In addition, contact based devices requires high degree of compliance from patients which is not always possible especially in case of unconscious and very young patients. The contact nature of a device also brings the issue of cleanliness. Recent advancements in computer vision and computing have enabled researchers to develop non-invasive systems.

The Optoelectronic Plethysmography System was the first non-invasive optical based system for pulmonary functional testing. The pioneering work was done by Ferrigno \& Pedotti [3] in 1985 and the system was called "The ELITE" (ELITE System; BTS, Milan, Italy). They used a digital video system and motion analyzer system to identify and track predefined markers placed on a person's torso. By tracking the markers they were able to construct a 3D model of the respiratory ribcage, abdomen ribcage and the abdomen. By monitoring the expansion and contraction of this 3D model they were able to quantify the change in volume which corresponds to measurement of breathing. The main problem with this system was its high error $( \pm 21.3 \%)$ when compared with the traditional Spirometer [4]. The error was caused by the assumption of a cubical geometry of chest wall shape.

Later many researchers modified this assumption to circumferential geometry to improve the accuracy of measurement [4-6]. The 86 markers method used by Cala et al. in 1996 [4] reduced the error of ELITE to less than $3.5 \%$. Cala also patented his method and renamed it to OptoElectronic Plethysmography system (OEP) (OEP System; BTS, Milan, Italy). The OEP measures the volume in three different regions of the chest and abdomen by tracking makers with up to eight cameras (four for front and four for back). A full three-dimensional shape of the trunk is constructed and volume is calculated using Divergence Theorem [7] also known as Gauss's theorem.

Cala et al. showed that there is a strong correlation between volume measured from OEP and volume measured from the Spirometer [4] both in quiet breathing as well as in different breathing manoeuvres. OEP has been also validated for a variety of applications such as intensive care patients [8], in infants [9] and during exercise in chronic obstructive pulmonary disease $[5,10,11]$. It has been also validated on subjects in prone and supine positions [12]. Although the concept of vision based Spirometry is validated, this system is not a non-contact system.

*Jahanzeb.Ahmad@uwe.ac.uk; +44-117-32-86807; www.uwe.ac.uk/et/mvl 
Aoki et al [13] in 2005 presented a triangulation based respiration monitoring and measurement method. The system was based on a fiber grating vision sensor, which is composed of a multiple slit light projector and a CCD camera. It projected 300 bright spots on the sleeping patient these projected points are then observed from the CCD camera and processed to generate $3 \mathrm{D}$ data. This system can capture and calculate volume at up to 5 frames per second using a triangulation method. Validation was performed by simultaneously measuring volume from 3D data and a Spirometer which showed high correlation of 0.96 . The system does however have a very low spatial and temporal resolution.

Recently a group of researchers from Cambridge University, Cambridge UK and PneumaCare Limited, Duxford UK have developed a new Non-Contact (marker-less) system for respiratory functional testing [14]. They use a structured light method [15] to capture a three-dimensional shape of patient's chest by using two digital cameras and a projector. Both cameras are placed in different but known positions and angles with respect to patient's chest. The patient is requested to wear a tightly fitting white T-shirt and then to sit on a firm back chair or lay down on mat in the supine position and breathe in a natural way. Edge detection methods are used to detect the corners. The corners are tracked and corresponded with images from both cameras to construct the three-dimensional shape of the scene. After removing unwanted data and filling the missing data points only the chest's three-dimensional shape is remaining. The volume of the given chest three-dimensional shape is calculated between the chest and the back of the chair or mat. Like OEP they also use Gauss's Theorem [7] for volume calculation. The validation of the system was performed on 40 healthy subjects and a strong correlation with Spirometer data was found. However the system still needs further validation on real patients. The current system features low spatial and temporal resolution and cannot handle changes in physical movement of the patient.

Photometric stereo was first introduced by Woodham in 1980 [16]. It recovers the local surface orientation of the object or scene by taking several images from the same view point but under different lighting conditions. The Light sources are some distance away from the scene with different directions. Each pixel at the same location within all the images is assumed to correspond to the same object point so there is no need to match features between images.

\section{Hardware Setup}

The experimental setup is composed of two monochrome digital cameras 1.4 Mega pixel each and eight light sources. The rig is composed of two identical sub-rigs each sub-rig has one camera and four light sources as shown in the Fig. 1. The Subject is standing in middle of rig wearing a white tight t-shirt and asked to do diaphragmatic breathing. Current light sources used are single wavelength blue light. Corresponding band pass filters are used to suppress the background light in the room.

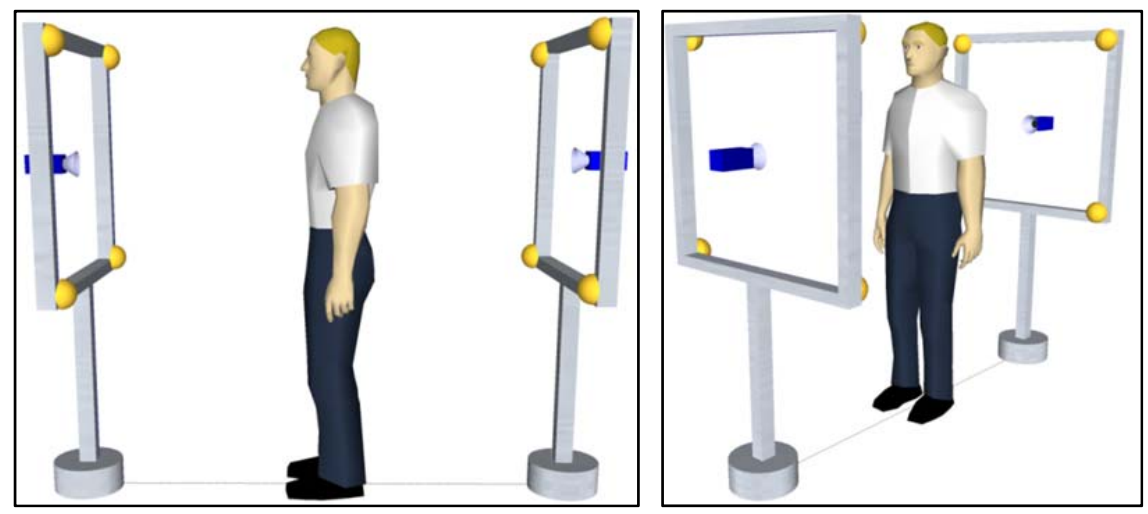

Fig. 1: Photometric stereo Rig with subject standing in middle of rig.

The system is designed so that it can run using an ordinary office desktop computer. Timing of the lighting and the camera trigger are controlled using an FPGA board [17]. Custom embedded software for FPGA was designed using VHDL. The cameras are directly connected with computer using Gigabit Ethernet cards. To get ground-truth data analog Pneumotachometers and transducers are used. The signal from transducer is digitized using a 12-bit analog to digital converter on a custom designed board and stored on a FPGA board. The overall block diagram of the Hardware setup is shown in Fig. 2. 


\section{Data Acquisition}

Images are captured in raw binary format and stored on a high speed solid state drive. Once the data acquisition is done the captured images are transferred on an ordinary hard disk for processing. A high speed FPGA based embedded system is designed to precisely control the timing for camera trigger and lighting. Currently the system is capable of capturing at 110 FPS without any image losses, for around 10 minutes. This restriction of 10 minutes can be reduced by using a bigger solid state drive. Custom board samples the Pneumotachometer data at the rate of 1000 samples per second. A typical Pneumotachometer volume (PV) signal is shown in Fig. 3.

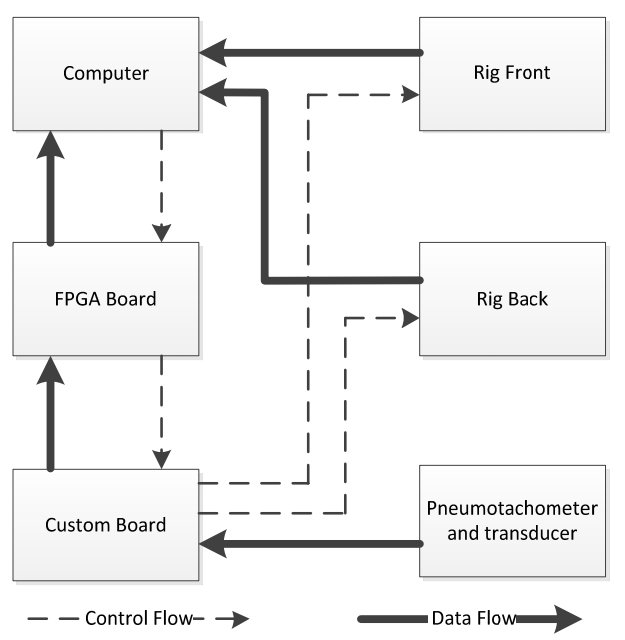

Fig. 2: Block diagram of Hardware. Dotted arrows represent flow of camera trigger and light source power flow. Solid arrows represent flow of raw images and signal obtained from Pneumotachometer.

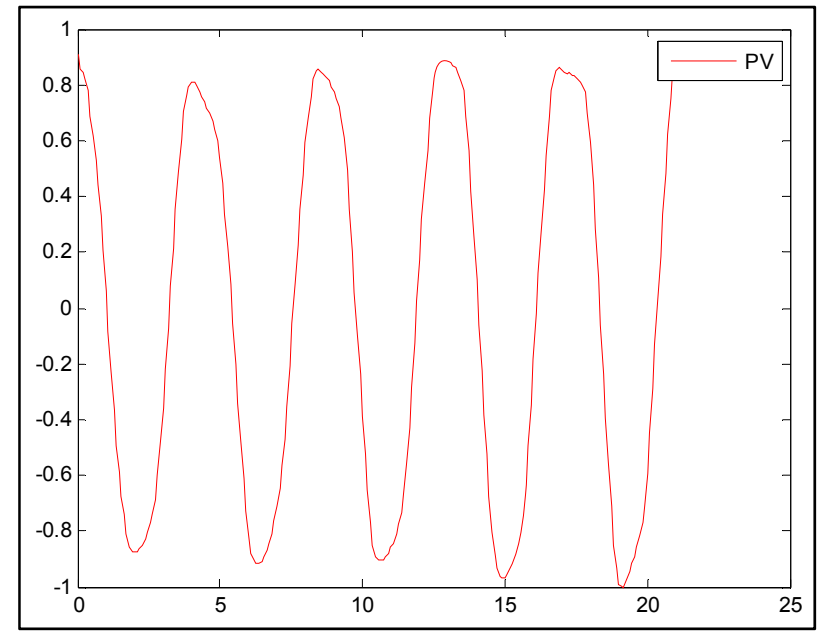

Fig. 3: Pneumotachometer volume (PV) signal obtained after digitization. Horizontal axis is time in seconds. Vertical scale is a normalized unit less representation of volume, to compare volume obtained from Pneumotachometer and Gradient.

To estimate surface gradient in Photometric Stereo requires at least three images taken from the same view point but with different light directions. In our setup we use five images to estimate surface gradient. Four images are taken by turning on one light source at a time as shown in Fig. 4(a) to Fig. 4(d). Light sources are placed at equal distance from each other to get homogenous light distribution among all four images as shown in Fig. 1. The fifth image is taken when no light source is turned on to get the background light intensity (noise) as shown in Fig. 4(e). As images are taking in unconstrained room environment a light on ceiling is visible in Fig. 4(e). This room noise is removed using background subtraction. By using a circular shift method we are able to generate 3D data (surface gradient) at $\sim 44 \mathrm{fps}$ for the whole torso. 


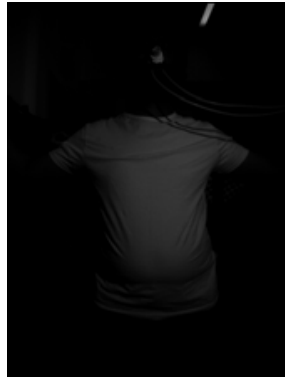

(a)

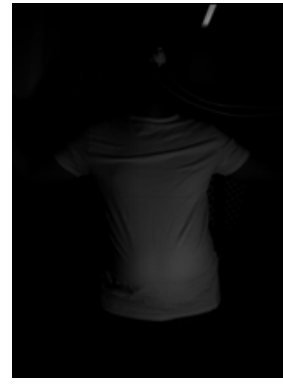

(b)

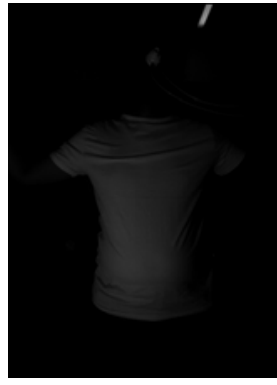

(c)

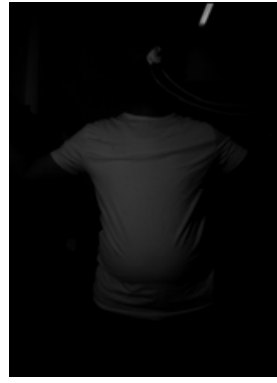

(d)

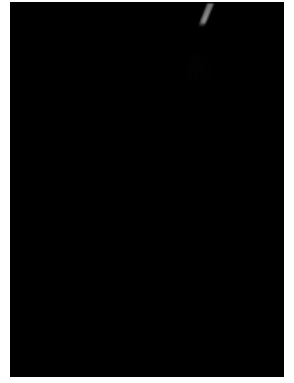

(e)

Fig. 4: Raw Images obtained from Front Rig. Image(a) when top-right light is turned on, image(b) when bottom-right light is turned on, image (c) when bottom-left light is turned on, image (d) when top-left light is turned on and image $(e)$ is when no light is turned on.

\section{Data processing}

Data is processed offline because of the high frame rate and spatial resolution of the images. The multiple stages of the processing are explained below.

\subsection{Torso segmentation}

To monitor chest and abdomen movement the torso is segmented from images as the whole image contains other parts of body. Average image Iave is obtained by removing background noise from all four images and then taking the average as shown in equation 1.

$$
\text { Iave }=\frac{1}{4} \sum_{i=0}^{3}\left(I m g_{i}-I b g\right)
$$

The average image is then converted into a Black and white binary image by applying adaptive thresholding [18]. To segment the torso from black and white image, connected components[19] with eight-connected neighbours are computed and the component with highest area is assumed to be torso as shown in Fig. 5(a). The torso is smoothed and access data (limbs) are removed to get the final mask of torso (Fig. 5(b)). This process is repeated for both front and back images independently.

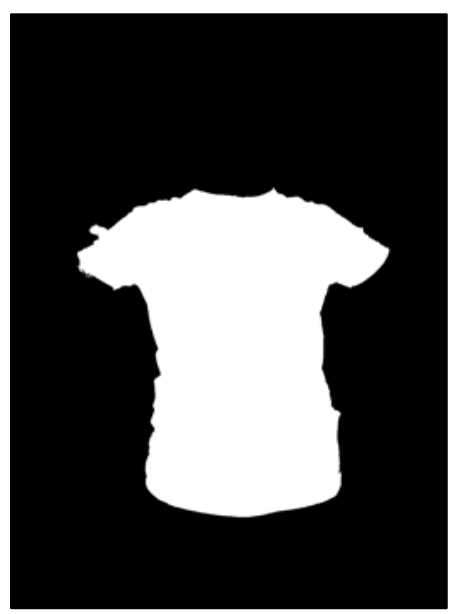

(a)

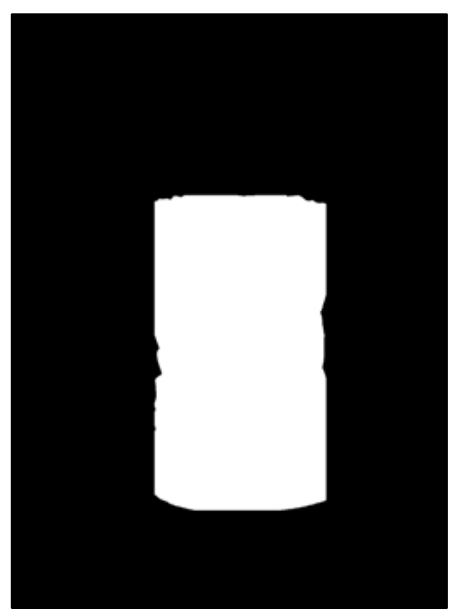

(b)

Fig. 5: image(a) obtained after segmenting component with highest area image(b) after removing unwanted regions of torso from image(a) 


\subsection{Surface Gradient calculations}

The mask generated in the section above is then applied to images before applying photometric stereo to calculate the surface gradients. Assuming the object surface is Lambertian, the Lambertian reflectance model can be used to calculate the surface gradients $p$ and $q$ by using Equation 2. $p$ is the gradient of the surface along $\mathrm{x}$-axis and $q$ is the gradient of the surface along $\mathrm{y}$-axis.

$$
I^{i}(x, y)=\rho(x, y) n(x, y) s^{i}
$$

$\mathrm{I}$ is image intensity, $\rho$ is the albedo of the scene and $s$ is the light source direction vector, where $\mathrm{i}=1$, 2,3 and 4.

$$
\begin{gathered}
\vec{I}(x, y)=\left(I^{1}(x, y), I^{2}(x, y), I^{3}(x, y), I^{4}(x, y),\right) \\
{[S]=\left(\vec{S}^{1}, \vec{S}^{2}, \vec{S}^{3}, \vec{S}^{4}\right)}
\end{gathered}
$$

$\vec{I}$ is the vector of the images $[S]$ is the matrix light vectors. Equation 2 can be rewritten in vector form by using equation 3 and 4 . By using the least square method $\vec{M}(x, y)$ can be calculated to calculate $\mathrm{p}$ and $\mathrm{q}$.

$$
\begin{gathered}
\vec{M}(x, y)=\left(s^{T} s\right)^{-1} s^{T} \vec{I}(x, y) \\
\vec{M}(x, y)=\left(m_{1}(x, y), m_{2}(x, y), m_{3}(x, y)\right) \\
p(x, y)=\frac{-m_{1}(x, y)}{m_{3}(x, y)} \\
q(x, y)=\frac{-m_{2}(x, y)}{m_{3}(x, y)}
\end{gathered}
$$

\subsection{Volume calculation from gradient}

The volume of the torso is represented as the total average gradient; we call it the gradient volume (GV). GV is calculated by adding the absolute average of $p$ and $q$ as shown in equation 9 for both front and back.

$$
G V=\left[\frac{1}{k} \sum\left(\mid p_{-} \text {front }|+| q_{-} \text {front } \mid\right)\right]+\left[\frac{1}{l} \sum\left(\mid p_{-} \text {back }|+| q_{-} \text {back } \mid\right)\right]
$$

$k$ and $I$ are the total no of valid pixels in the front and back images respectively. To compare GV with volume obtained from Pneumotachometer normalization is applied on both signals using equation 10.

$$
V=\frac{V}{\max \left(\left|V-\left[\frac{1}{t} \Sigma V\right]\right|\right)}
$$

Here $t$ represents total values in the temporal domain. A typical $G V$ signal obtained from the method explained above and normalized by equation 10 is shown in Figure 6. 


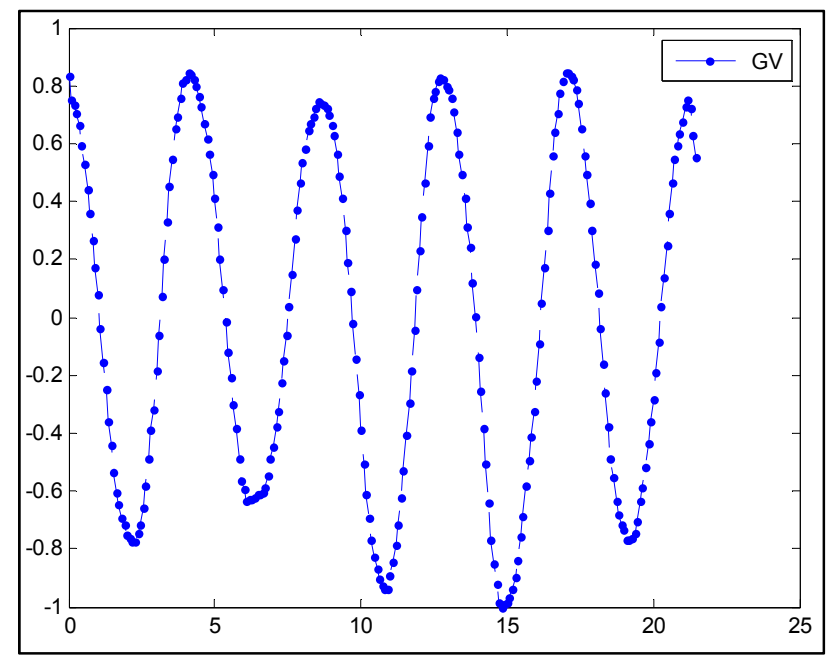

Fig. 6: Volume obtained from surface gradient (GV). Horizontal axis is time in seconds. Vertical scale is a normalized unit less representation of volume, to compare volume obtained from Pneumotachometer and Gradient.

\section{Results}

High correlation (0.98) between Gradient volume and Pneumotachometer volume was found as shown in Fig. 7.

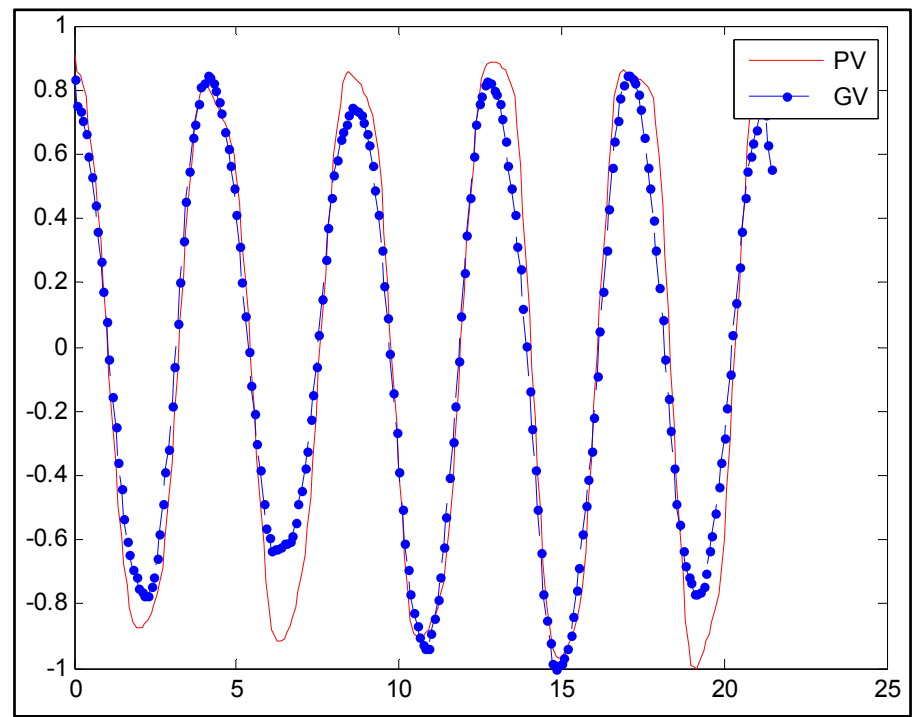

Fig. 7: Pneumotachometer Volume (PV) and Gradient Volume (GV) (combined front and back). Horizontal axis is time in seconds. Vertical scale is a normalized unit less representation of volume, to compare volume obtained from Pneumotachometer and Gradient.

Analysis on the front and back has been performed independently, which suggests that the correlation of the back Gradient volume is negatively correlated to the Pneumotachometer volume and front Gradient volume is positively correlated to Pneumotachometer volume as shown in Fig. 8. Front GV shows high correlation with PV. But when back GV which is weakly and negatively correlated with PV is added to front GV it yields higher correlation as compared to front GV only. This phenomenon is shown in the Fig. 8 as well as in table 1. 
Table 1: Gradient Volume analyis and comparision of front, back and combined.

\begin{tabular}{|l|c|}
\hline & Correlation \\
\hline Front GV & 0.93 \\
\hline Back GV & -0.54 \\
\hline Combined GV & 0.98 \\
\hline
\end{tabular}
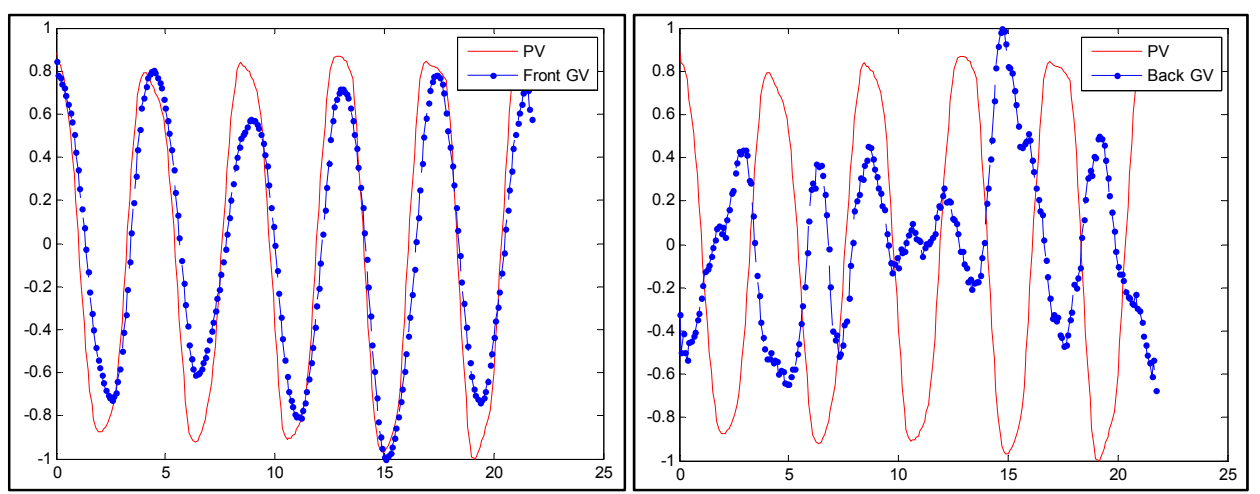

Fig. 8: Left figure front Gradient Volume (GV) and Pneumotachometer Volume (PV) plot, Right figure back GV and PV plot. Horizontal axis is time in seconds. Vertical scale is a normalized unit less representation of volume, to compare volume obtained from Pneumotachometer and Gradient.

The system is also quite tolerant to the subject's position and orientation in the rig. Fig. 9(a) and Fig. 9(b) shows the correlation between volume obtained from Pneumotachometer and gradients while the subject is standing in different locations on the rig.

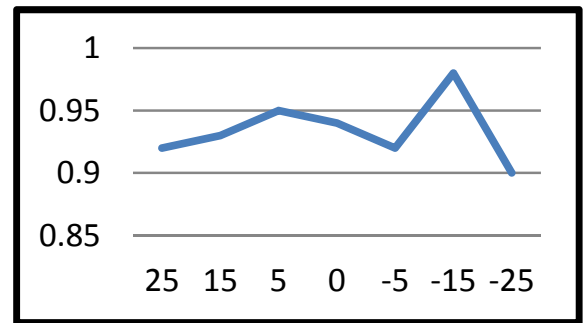

(a)

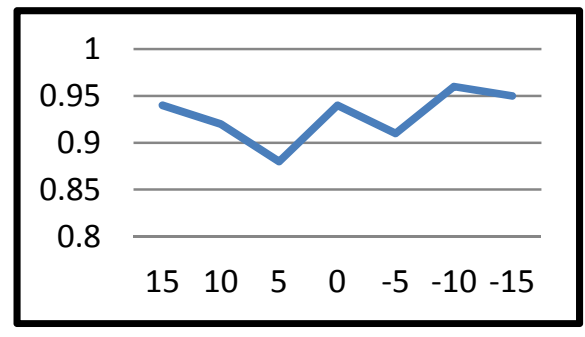

(b)

Fig. 9: Subject displacement chart in Sagittal and Coronal plane. Vertical axis is the correlation between Gradient volume and Pneumotachometer volume. Horizontal axis is the displacement of subject from centre of rig in Sagittal and Coronal plane in centimetres.

\section{Conclusion and future work}

We have proposed a Novel non-contact device to perform PFT on patients of any age. The unique features of the device are that it does not need any calibration or registration and it generates at least 2000 times higher resolution data compared to similar systems. As the system is based on off the shelf and cheap components it is relatively easy and cheap to make. High resolution images can be used for regional analysis of the torso. In future we are planning to use the device for a range of chest and back bone problems.

\section{References}

1. E. Schneider et al., (2006): "Comparison of tolerance of facemask vs. mouthpiece for non-invasive ventilation.," Anaesthesia, vol. 61, no. 1, pp. 20-3.

2. R. Gilbert, J. H. Auchincloss, J. Brodsky, and W. Boden, (1972): "Changes in tidal volume, frequency, and ventilation induced by their measurement," J Appl Physiol, vol. 33, no. 2, pp. 252-254.

3. G. Ferrigno and A. Pedotti,(1985): "ELITE: A Dedicated Hardware System for Movement Analysis Via Real-Time TV Signal Processing," no. 11, pp. 943-950. 
4. S. J. Cala et al., (1996): "Chest wall and lung volume estimation by optical reflectance motion analysis.," Journal of applied physiology, vol. 81, no. 6, pp. 2680-9.

5. A. Aliverti et al.,(2005): "Effect of salbutamol on lung function and chest wall volumes at rest and during exercise in COPD.," Thorax, vol. 60, no. 11, pp. 916-24.

6. N. A. Borghese and G. Ferrigno, (1990): "An algorithm for 3-D automatic movement detection by means of standard TV cameras.," IEEE transactions on bio-medical engineering, vol. 37, no. 12, pp. $1221-5$.

7. E. W. Weisstein, "Divergence Theorem," MathWorld --A Wolfram Web Resource (accessed 1 May 2012) : http://mathworld.wolfram.com/DivergenceTheorem.html

8. A. Aliverti, R. Dellaca, P. Pelosi, D. Chiumello, A. Pedotti, and L. Gattinoni,(2000): "Optoelectronic plethysmography in intensive care patients," American Journal of Respiratory and Critical Care Medicine, vol. 161, no. 5, p. 1546.

9. R. L. Dellaca', M. L. Ventura, E. Zannin, M. Natile, A. Pedotti, and P. Tagliabue,(2010): "Measurement of total and compartmental lung volume changes in newborns by optoelectronic plethysmography.," Pediatric research, vol. 67, no. 1, pp. 11-6.

10. A. Aliverti, (2004): "Regional chest wall volumes during exercise in chronic obstructive pulmonary disease," Thorax, vol. 59, no. 3, pp. 210-216.

11. O. Georgiadou et al., (2007): "Effects of rehabilitation on chest wall volume regulation during exercise in COPD patients.," The European respiratory journal: official journal of the European Society for Clinical Respiratory Physiology, vol. 29, no. 2, pp. 284-91.

12. A. Aliverti, R. Dellacà, P. Pelosi, D. Chiumello, L. Gattinoni, and A. Pedotti,(2001) "Compartmental Analysis of Breathing in the Supine and Prone Positions by Optoelectronic Plethysmography," Annals of Biomedical Engineering, vol. 29, no. 1, pp. 60-70.

13. H. Aoki, K. Koshiji, H. Nakamura, Y. Takemura, and M. Nakajima, (2005): "Study on respiration monitoring method using near-infrared multiple slit-lights projection," in IEEE International Symposium on Micro-NanoMechatronics and Human Science, pp. 291-296.

14. W. de Boer et al., (2010): "SLP: A Zero-Contact Non-Invasive Method for Pulmonary Function Testing," Procedings of the British Machine Vision Conference 2010, pp. 85.1-85.12.

15. L. Zhang, B. Curless, and S. M. Seitz, (2002): "Rapid shape acquisition using color structured light and multi-pass dynamic programming," in First International Symposium on 3D Data Processing Visualization and Transmission, pp. 24-36.

16. R. J. Woodham, (1980): "Photometric method for determining surface orientation from multiple images," Optical Engineering, vol. 19, no. 1, pp. 139-144.

17. Orange Tree Technologies Ltd "ZestSC1 FPGA board," (accessed 1 May 2012): http://www.orangetreetech.com/fpga board zestsc1.html.

18. N. Otsu,(1979): "A Threshold Selection Method from Gray-Level Histograms," IEEE Transactions On Systems Man And Cybernetics, vol. 9, no. 1, pp. 62-66.

19. B. Horn, (1986): "Robot vision," MIT Press. 